\title{
RATIONAL CHEBYSHEV APPROXIMATION TO CERTAIN ENTIRE FUNCTIONS OF ZERO ORDER ON THE POSITIVE REAL AXIS. II
}

\author{
A. R. REDDY
}

ABSTRACT. A sufficient condition is given on the growth of certain entire functions which guarantee a certain rate of convergence of the error in approximating reciprocals of a class of entire functions by reciprocals of polynomials under the uniform norm on the positive real axis.

Recently we proved

THEOREM 1 [1, THEOREM 7]. Let $f(z)=\sum_{k=0}^{\infty} a_{k} z^{k}, a_{0}>0$ and $a_{k}$ $\geqslant 0(k \geqslant 1)$ be an entire function of zero order satisfying the assumptions that

$$
1<\limsup _{r \rightarrow \infty} \frac{\log \log M(r)}{\log \log r}=\Lambda+1=\rho_{l}<\infty
$$

and

$$
0<\liminf _{r \rightarrow \infty} \frac{\log M(r)}{(\log r)^{\Lambda+1}}=b_{l}, \quad \limsup _{r \rightarrow \infty} \frac{\log M(r)}{(\log r)^{\Lambda+1}}=B_{l}<\infty
$$

where $M(r)=\max _{|z|=r}|f(z)|$. Then there exists a sequence of polynomials $\left\{P_{n}(x)\right\}_{n=0}^{\infty}$ for which

$$
\lim _{n \rightarrow \infty}\left\{\left\|\frac{1}{f(x)}-\frac{1}{P_{n}(x)}\right\|_{L_{\infty}[0, \infty)}\right\}^{1 / n}=0 .
$$

One sees that (2) implies the limit in (1), for suppose, to the contrary,

$$
\begin{aligned}
0 & <\liminf _{r \rightarrow \infty} \frac{\log \log M(r)}{\log \log r}=\sigma<\Lambda+1 \\
& =\limsup _{r \rightarrow \infty} \frac{\log \log M(r)}{\log \log r}<\infty .
\end{aligned}
$$

Then for each $\epsilon>0$, there is a sequence of values of $r$ for which

$$
\log M(r) \leqslant(\log r)^{\sigma+\epsilon}
$$

From (4) we obtain

Received by the editors August 27, 1974.

AMS (MOS) subject classifications (1970). Primary 41A20.

Key words and phrases. Rational approximation, zero order, positive real axis. 


$$
(\log M(r)) /(\log r)^{\Lambda+1} \leqslant(\log r)^{\sigma+\epsilon} /(\log r)^{\Lambda+1},
$$

and we may choose $\epsilon$ so that $\sigma+\epsilon<\Lambda+1$. Then clearly we have

$$
\liminf _{r \rightarrow \infty} \frac{\log M(r)}{(\log r)^{\Lambda+1}}=b_{l}=0,
$$

which contradicts (2).

On the other hand the limit in (1) may not imply (2). For example let

$$
\begin{aligned}
& f_{1}(z)=1+\sum_{n=2}^{\infty} \frac{z^{n}}{\exp \left[(n \log n)^{2}\right]}, \\
& f_{2}(z)=1+\sum_{n=2}^{\infty} \frac{z^{n}}{\exp \left[(n / \log n)^{2}\right]} .
\end{aligned}
$$

It is easy to calculate for

$$
f_{1}(z), \quad \Lambda=1=\sigma-1, \quad B_{l}=0, \quad f_{2}(z), \quad \Lambda=1=\sigma-1, \quad B_{l}=\infty .
$$

Hence it is natural to ask whether one can get (3) under the only assumption

$$
0<\lim _{r \rightarrow \infty} \frac{\log \log M(r)}{\log \log r}=\Lambda+1<\infty .
$$

In fact we prove the more general

THEOREM 2. Let $f(z)=\sum_{k=0}^{\infty} a_{k} z^{k}, a_{0}>0, a_{k} \geqslant 0(k \geqslant 1)$ be an entire function of zero order satisfying the assumption that

$$
0<\Lambda<\liminf _{r \rightarrow \infty} \frac{\log \log M(r)}{\log \log r}=\sigma<\infty .
$$

Then there exists a sequence of polynomials $\left\{P_{n}(x)\right\}_{n=0}^{\infty}$ for which

$$
\lim _{n \rightarrow \infty}\left\{\left\|\frac{1}{f(x)}-\frac{1}{P_{n}(x)}\right\|_{L_{\infty}[0, \infty)}\right\}^{1 / n}=0 .
$$

REMARK. The method used here is much simpler than the one used in [1]. Proof. By assumption

$$
\limsup _{r \rightarrow \infty} \frac{\log \log M(r)}{\log \log r}=\Lambda+1 .
$$

Then it is known [2, Theorems 1 and 3] that

$$
\limsup _{n \rightarrow \infty} \frac{\log n}{\log \left\{n^{-1} \log \left|a_{n}\right|^{-1}\right\}}=\Lambda \text {. }
$$

From (6) it is easy to get for each $\epsilon>0$ there is an $n_{0}$ depending on $\epsilon$ such that for all $n \geqslant n_{0}(\epsilon)$,

$$
\left|a_{n}\right| \leqslant \exp \left(-n^{1+1 /(\Lambda+\epsilon)}\right) .
$$


Let $S_{n}(z)=\sum_{k=0}^{n} a_{k} z^{k}$; then clearly for $0 \leqslant x \leqslant r$,

$$
0 \leqslant \frac{1}{S_{n}(x)}-\frac{1}{f(x)} \leqslant \frac{f(x)-S_{n}(x)}{f(x) S_{n}(x)} \leqslant a_{0}^{-2} \sum_{k=n+1}^{\infty} a_{k} r^{k} .
$$

From (7) and (8) we get, for $0 \leqslant x \leqslant r$,

\section{Choose}

$$
\begin{aligned}
0 & \leqslant \frac{1}{S_{n}(x)}-\frac{1}{f(x)} \leqslant a_{0}^{-2} \sum_{k=n+1}^{\infty} \exp \left(-k^{1+(1 /(\Lambda+\epsilon)}+k \log r\right) \\
& =a_{0}^{-2} \sum_{k=n+1}^{\infty} \exp \left[\left(-n^{(1 /(\Lambda+\epsilon))}+\log r\right) k\right] .
\end{aligned}
$$

$$
2 \log r=n^{(1 /(\Lambda+\epsilon))} .
$$

Then we have from (9) that

$$
\begin{aligned}
0 & \leqslant \frac{1}{S_{n}(x)}-\frac{1}{f(x)} \leqslant a_{0}^{-2} \sum_{k=\Lambda+1}^{\infty} \exp \left[\left(\frac{-n^{(1 /(\Lambda+\epsilon))}}{2}\right) k\right] \\
& \leqslant C_{1} \exp \left(-n^{1+1 /(\Lambda+\epsilon)} C_{2}\right)
\end{aligned}
$$

where $C_{1}, C_{2}$ are some constants. On the other hand for all $x \geqslant r$, we get

$$
0 \leqslant \frac{1}{S_{n}(x)}-\frac{1}{f(x)} \leqslant \frac{1}{S_{n}(x)} \leqslant \frac{1}{S_{n}(r)}=\frac{1}{f(r)-\sum_{k=n+1}^{\infty} a_{k} r^{k}}
$$

By assumption

$$
0<\liminf _{r \rightarrow \infty} \frac{\log \log M(r)}{\log \log r}=\sigma<\infty .
$$

From this we get for all $r \geqslant r_{0}(\epsilon)$,

$$
f(r)=M(r) \geqslant \exp \left((\log r)^{\sigma-\epsilon}\right) .
$$

On the other hand we know from (8) and (11) that

$$
\sum_{k=n+1}^{\infty} a_{k} r^{k} \leqslant \exp \left(-C_{2} n^{(1 /(\Lambda+\epsilon))}\right)
$$

Hence from (12), (13) and (14) we get, with a little manipulation, for all $x \geqslant r$,

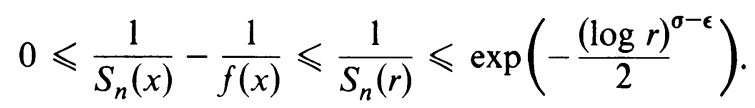

From (15) and (10) we obtain for all $x \geqslant r$,

$$
0 \leqslant \frac{1}{S_{n}(x)}-\frac{1}{f(x)} \leqslant \frac{1}{S_{n}(r)} \leqslant \exp \left(-C_{3}(n)^{(\boldsymbol{\sigma}-\epsilon) /(\Lambda+\epsilon)}\right),
$$

where $C_{3}$ is a suitable constant. By choosing $\epsilon$ such that $\sigma-\epsilon>\Lambda+\epsilon$, we 
get from (11) and (16) the required result, i.e.

$$
\lim _{n \rightarrow \infty}\left\{\left\|\frac{1}{f(x)}-\frac{1}{P_{n}(x)}\right\|_{L_{\infty}[0, \infty)}\right\}^{1 / n}=0 .
$$

I would like to thank the referee for his suggestions.

\section{REFERENCES}

1. G. Meinardus, A. R. Reddy, G. D. Taylor and R. S. Varga, Converse theorems and extensions in Chebyshev rational approximation to certain entire functions in $[0,+\infty)$, Trans. Amer. Math. Soc. 170 (1972), 171-185. MR 46 \# 9603.

2. A. R. Reddy, On entire Dirichlet series of zero order, Tôhoku Math. J. (2) 18 (1966), 144 155. MR 35 \#5614.

Department of Mathematics, Michigan State University, East Lansing, Michigan 48823

Current address: Department of Mathematics, Yeshiva University, New York, New York 10033 